\title{
Rupture Uterus in Pregnancy with Didelphys Uterus A Rare Case Report
}

\author{
Shashi Suthar, Rekha Choudhary, Shaifali Dadhich \\ Senior Resident, Department of Obstetrics and Gynecology, SP Medical College and Attached Group of Hospital, Bikaner, Rajasthan, India
}

Correspondence: Rekha Choudhary, Senior Resident, Department of Obstetrics and Gynecology, SP Medical College and Attached Group of Hospitals, Bikaner, Rajasthan, India, Phone: +91-9461245850, e-mail: rekhatappu@gmail.com

\section{ABSTRACT}

Uterus didelphys is rare and sometimes not even diagnosed. We report a case of didelphys uterus in a 26-year-old pregnant lady who previously had three successful pregnancies with previous one C-section and remained undiagnosed till she presented to us as ruptured uterus. This case report aimed to summarize the clinical characteristics and perinatal outcome of VBAC in pregnancy with didelphys uterus and history of previous cesarean section.

Keywords: Rupture uterus, Didelphys uterus, Previous cesarean section, Vaginal birth after cesarean section (VBAC).

\section{INTRODUCTION}

The prevalence rate of uterine malformations in general fertile population is 0.001 to $10 \%$. Fusion of the two müllerian ducts and the establishment of the vaginal canal are usually completed between the 10th and 17th week of intrauterine development. Sometimes, however, the müllerian ducts do not join completely. Several degrees of duplication of the uterus are possible, ranging from a complete duplication of the uterus, cervix and vaginal canal. Instead, each one develops into a separate cavity. This condition is called double uterus (uterus didelphys). Some women with a double uterus also have a duplicate or divided vagina. Ruptured uterus in pregnancy is rare and often leads to catastrophic complication with a high incidence of fetal and maternal morbidity and mortality.

\section{CASE REPORT}

A 26-year-old unbooked, G4P $\mathrm{P}^{3+0+0+3}$ housewife was rushed to our hospital at 39 weeks of gestation with a history of severe generalized abdominal pain and weakness of about 4 hours prior to presentation. She was not registered with her antenatal care attendance. The index pregnancy was marked with persistent abdominal pain which started in her 2nd trimester for which ultrasound scan was ordered. However, this was not done. In previous obstetric history, she had three previous live babies. First baby was full-term normal delivered at home, 2nd baby was delivered by the cesarean section, but there was no record available regarding indication and procedure, 3rd baby was again full-term delivered at home and uneventful. At the time of admission she was very pale, afebrile, normotensive with tachycardia. On per abdominal examination tenderness was present all over the abdomen, uterine contour was absent, fetal parts were palpable superficially and fetal heart sound was not localized on auscultation. Bleeding was present per vaginally. Two cervices were felt to finger on per vaginal examination.
Right cervix admitted two finger and presenting part felt high up and left cervix admitted just tip of the finger and a septum felt in between two cervical openings. A tentative diagnosis of spontaneous rupture of the gravid uterus was made. On ultrasonography she was diagnosed as single IUD of 37 weeks gestations size with ruptured uterus. She was resuscitated with 2 liters of normal saline and two units of fresh whole blood and immediate laparatomy was done. Findings at laparatomy showed massive hemoperitoneum, a fresh stillbirth lying freely in the peritoneal cavity (weight $=3 \mathrm{~kg}$ ), ragged uterine tissues with placenta lying freely in the peritoneal cavity. Patient was bleeding freely from the edges of the ruptured uterus and no other bleeding surface demonstrable. On examination, uterus didelphys was present. Pregnancy was in right uterus and same was ruptured at previous scar site (Fig. 1). Repair of the uterus along with sterilization was done after taking the consent. Postoperative period was uneventful. Before discharge, USG pelvis was done to rule out pregnancy in left uterus.

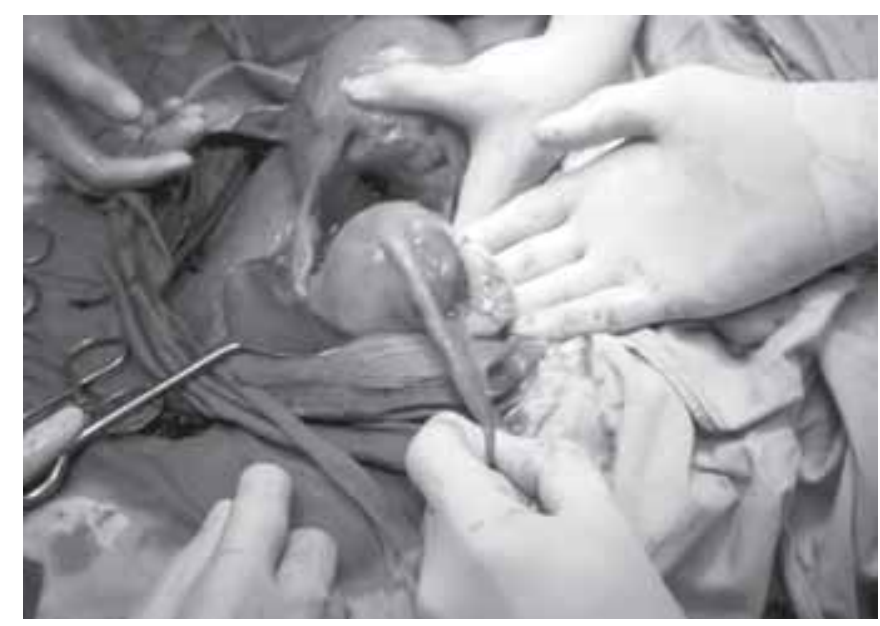

Fig. 1: Ruptured uterus at site of previous scar in a pregnancy with didelphys uterus 


\section{DISCUSSION}

According to one estimate, didelphys uterus occurs in 0.1 to $0.5 \%$ healthy fertile population. The double uterus (didelphys) has a poor reproductive outcome with a 20 to $30 \%$ chance of carrying pregnancy to term. Normal pregnancies can occur in patients with müllerian duct anomalies, but obstetric complications such as spontaneous abortion, stillbirth and preterm birth are frequent. Didelphys uterus should always be considered in cases of severe dysmenorrhea and chronic pelvic pain; of the failure of intrauterine contraceptive devices; of a symptomatic or asymptomatic pelvic mass that is inseparable from the uterus.

Incidence of overall uterine rupture rate was one in 1,536 pregnancies $(0.07 \%)$. The uterine rupture rate for women undergoing a TOL after previous cesarean delivery was $0.39 \%$ compared with $0.16 \%$ for patients undergoing elective repeat cesarean delivery. A study by Lydon-Rochelle et al showed that the uterine rupture rate among 10,789 women with a single previous cesarean delivery who labored spontaneously during a subsequent singleton pregnancy was $0.52 \%$ and increased relative risk (RR) of 3.3 (95\% CI, 1.8-6.0) for women who labor spontaneously compared with women who undergo elective repeat cesarean delivery. ${ }^{1}$ As a rule, the time available for successful intervention after frank uterine rupture and before the onset of major fetal morbidity is only 10 to 37 minutes. The vast majority of uterine ruptures occurs in women who have uterine scars, most of which are the result of previous cesarean deliveries. A single cesarean scar increases the overall rupture rate to $0.5 \%$, with the rate for women with two or more cesarean scars increasing to $2 \%$. Other subgroups of women who are at increased risk for uterine rupture are those who have a previous single-layer hysterotomy closure, a short interpregnancy interval after a previous cesarean delivery, a congenital uterine anomaly, a macrosomic fetus, prostaglandin exposure, and a failed previous trial of a vaginal delivery. Erez $\mathrm{O}$ reported that the rate of vaginal birth after cesarean section was significantly lower among patients with müllerian anomalies than in patients with normal uterus, 37.6\% (62/165) vs 50.7\% (2740/5406), respectively $(p=0.0009) .^{2}$ Nahum reported that uterine anomalies were identified in one in 594 fertile women (0.17\%) and in one in 29 infertile women (3.5\%). This difference was statistically significant $(\mathrm{p}<0.00001)$. The prevalence of uterine anomalies in the general population was one in 201 women (0.50\%). Their distribution was $7 \%$ arcuate, $34 \%$ septate, $39 \%$ bicornuate, $11 \%$ didelphic, 5\% unicornuate, and $4 \%$ hypoplastic/aplastic/solid and other forms. In such cases, the walls of the abnormal uteri tend to become abnormally thin as pregnancies advance, and the thickness can be inconsistent over different aspects of the myometrium. ${ }^{3}$ Ravasia et al reported an $8 \%$ incidence of uterine rupture (2 of 25) in women with congenitally malformed uteri compared with $0.61 \%$ (11 of $1,788)$ in those with normal uteri $(p=0.013)$ who were attempting VBAC. ${ }^{4}$

\section{REFERENCES}

1. Lydon-Rochelle M, Holt VL, Easterling TR, Martin DP. Risk of uterine rupture during labor among women with a prior cesarean delivery. N Engl J Med. Jul 5 2001;345(1):3-8.

2. Erez O, Dukler D, Novack L, Rozen A, Zolotnik L. Trial of labor and vaginal birth after cesarean section in patients with uterine Müllerian anomalies: A population-based study. Am J Obstet Gynecol 2007;196:537.

3. Nahum GG. Uterine anomalies, induction of labor, and uterine rupture. Obstet Gynecol. Nov 2005;106(5):1150-52.

4. Ravasia DJ, Brain PH, Pollard JK. Incidence of uterine rupture among women with mullerian duct anomalies who attempt vaginal birth after cesarean delivery. Am J Obstet Gynecol Oct 1999;181(4):877-81. 